\title{
Cytotoxicity Studies of the Chemical Constituents from Freshwater Algae Chara baltica
}

\author{
V. B. TATIPAMULA* ${ }^{*}$ K. N. KILLARI ${ }^{1}$, K. PRASAD ${ }^{1}$, G. S. N. K. RAO, M. R. TALLURI ${ }^{2}$, SHYAMALA VANTAKU ${ }^{3}$, DEEPIKA \\ BILAKANTI ${ }^{4}$ AND NALLAPATY SRILAKSHMI
}

K L College of Pharmacy, Koneru Lakshmaiah Education Foundation, Vaddeswaram-522 502, ${ }^{1}$ Shri Vishnu College of Pharmacy, Bhimavarm-534 202, ${ }^{2}$ Anacipher Clinical Research Organization, Hyderabad-500 013, ${ }^{3}$ Government Polytechnic College, Madhavadhara, Visakhapatnam-530 007, ${ }^{4}$ Srikrupa Institute of Pharmaceutical Sciences, Velikatta, Kondapak, Siddipet-502 277, India

\section{Tatipamula et al.: Anticancer activity of Chara baltica}

\begin{abstract}
For the first time, 89 chemical constituents were identified by gas chromatography-mass spectrometry analysis of ethanol extract of Clathria baltica. The chemical examination of ethanol extract of Clathria baltica yielded three known metabolites, 1, 2 and 3, for first time from the marine algae Clathria baltica. All three metabolites and the ethanol extract of Clathria baltica were screened against cancer cell lines, MCF-7, DLD-1, HeLa, FADU, A549, and SKOV3, and one normal human cell line using sulforhodamine B assay and doxorubicin as the standard. Among all isolates, compound 3 showed significant growth inhibition of MCF-7, DLD-1 and FADU with $\mathrm{IC}_{50}$ values of $26.5,15.5$ and $16.5 \mu \mathrm{g} / \mathrm{ml}$, respectively. Whereas, all the metabolites and the extract exerted lower growth inhibitory effect on the normal human cell line tested. This is the first in vitro gas chromatography-mass spectrometry analysis, as well as, cytotoxicity report on marine algae Clathria baltica.
\end{abstract}

Key words: GC-MS analysis, isolation, characterization, anticancer activity, sulforhodamine B assay

Globally, cancer still remains an aggressive killer that severely effecting the human population ${ }^{[1]}$. According to the report of the International Agency for Research on Cancer (IARC), it was documented that within 5 y of diagnosis - 9.6 million cancer deaths from 33.7 million people living with cancer has taken place and 18.1 million new cancer cases were reported in 2018 across the world ${ }^{[2]}$. In addition, the IARC is also estimated that about 26 million new cancer cases and 17 million cancer deaths per year may be recorded by $2030^{[3]}$. In addition, the present therapies for cancer include chemotherapy and radiotherapy, which cause lot of strain to patients and ultimately damage their health ${ }^{[4]}$. Hence, researchers are focussing on developing new, safer and effective drugs to treat cancer.

From the dawn of ancient medicine, natural products have been used to treat many deadly diseases like cancer. In addition, from the past $30 \mathrm{y}$, natural products have received great attention in attaining potential as well as novel cancer therapeutic agents ${ }^{[5,6]}$. Moreover, the mechanism of action of inhibiting various stages

*Address for correspondence E-mail: vinaybharadwajt@gmail.com

September-October 2019 of tumorigenesis by most of the natural products were also well-documented ${ }^{[6]}$.

In folklore, marine algae (seaweeds) are used as food, feed, fuel and livelihood in Asian countries. Based on the pigmentation, marine algae are classified as red, brown and green algae. From the past few decades, marine algae have been highly acknowledged to possess noticeable pharmacological activities, which include antineoplastic, antimicrobial and antiviral activities due to their specific functional compounds (which are not available in other plants $)^{[7-10]}$. Particularly, marine algae of Chara genus (family: Characeae- charophyte green algae) reported to possess and antioxidant enzyme, allelophatic activities and lipid peroxidation capabilities along with high levels of photosynthetic pigment content ${ }^{[11]}$. Earlier reports from our laboratory showed phytochemical analysis along with antioxidant

This is an open access article distributed under the terms of the Creative Commons Attribution-NonCommercial-ShareAlike 3.0 License, which allows others to remix, tweak, and build upon the work non-commercially, as long as the author is credited and the new creations are licensed under the identical terms

Accepted 19 July 2019

Revised 03 April 2019

Received 22 January 2019

Indian J Pharm Sci 2019;81(5):815-823 
activities of marine algae Chara baltica ${ }^{[12]}$. Till date, complete chemical and pharmacological evaluation has not been reported on C. baltica. Based on the aforementioned properties of Chara genus, as well as, C. baltica, C. baltica specimens (in July 2018) were subjected to gas chromatography-mass spectrometry (GC-MS) analysis, chemical and cytotoxicity evaluation, which are reported in this research communication.

\section{MATERIALS AND METHODS}

The specimens of seaweed Chara baltica was collected near Korangi coast, Kakinada, Andhra Pradesh, India at a depth of 8-10 ft $\left(16^{\circ} 80^{\prime} \mathrm{N}\right.$ and $82^{\circ} 08^{\prime} \mathrm{E}$ with $3 \mathrm{~m}$ elevation) on 3 July, 2018. The seaweed was authenticated in the Botany Department, Andhra University, Visakhapatnam, India. A voucher specimen was deposited in the Marine Organisms Museum in the University College of Pharmaceutical Sciences, Andhra University, Visakhapatnam, India (No. AU-SW-2018-947). All chemicals used in the present study were of analytical grade. Streptozotocin was from Himedia Laboratories Pvt. Ltd. (Mumbai, India); glibenclamide from the Aventis Pharma Ltd. (Mumbai, India); and rat feed from the Hindustan Lever Ltd. (Mumbai, India).

\section{Extraction and isolation:}

The seaweed was cleansed from extraneous substance and stored in ethanol-water (9:1) at the site of collection. The crude plant material (100 g) was extracted at least thrice with ethanol-water and concentrated at a reduced pressure and the combined extracts were lyophilized to obtain ethanol extract of $C$. baltica; as a reddish brown gummy residue $(3.25 \mathrm{~g})$. About $2 \mathrm{~g}$ of ethanol extract was subjected to column chromatography, afforded three fractions. Fraction I $(510 \mathrm{mg})$ and fraction II ( $800 \mathrm{mg}$ ) were subjected to repeated column chromatography using hexane and ethyl acetate as a solvent system yielded $1(240 \mathrm{mg})$ as a colourless liquid and $2(330 \mathrm{mg})$ as a pale greenish liquid, respectively. Fraction III (400 mg) were purified by recrystallization technique using hexane and acetone as solvents yielded $3(200 \mathrm{mg})$ as a greenish crystals.

Compound 1 (dihydrofuran-2,5-dione), colorless crystals, molecular formula: $\mathrm{C}_{4} \mathrm{H}_{4} \mathrm{O}_{3}$; yield: $240 \mathrm{mg}$; $\mathrm{R}_{\mathrm{f}}: 0.7$ (hexane:ethyl acetate, 1:9); ${ }^{1} \mathrm{H} \mathrm{NMR}\left(\mathrm{CDCl}_{3}\right.$, $400 \mathrm{MHz}) \delta 4.00\left(4 \mathrm{H}, \mathrm{s}, 2,3-\mathrm{CH}_{2}\right) ;{ }^{13} \mathrm{C} \mathrm{NMR}\left(\mathrm{CDCl}_{3}\right.$, $400 \mathrm{MHz}) \delta 22.16$ (C-2/C-3), 170.24 (C-1/C-4); LCMS $m / z: 100$.
Compound 2 (3-hydroxy-4,4-dimethyldihydrofuran2(3H)-one), pale greenish liquid, $\mathrm{C}_{6} \mathrm{H}_{10} \mathrm{O}_{3}$; yield: $330 \mathrm{mg} ; \mathrm{R}_{\mathrm{f}}: 0.6$ (hexane:ethyl acetate, 1:4); ${ }^{1} \mathrm{H}$ NMR $\left(\mathrm{CDCl}_{3}, 400 \mathrm{MHz}\right) \delta 1.00\left(6 \mathrm{H}, \mathrm{s}, 5,6-\mathrm{CH}_{3}\right), 2.31(1 \mathrm{H}$, s, 2-CH), 3.86-3.88 (1H, d, $\left.J=8 \mathrm{~Hz}, 4 \mathrm{a}-\mathrm{CH}_{2}\right), 4.04-$ $4.06\left(1 \mathrm{H}, \mathrm{d}, J=8 \mathrm{~Hz}, 4 \mathrm{a}-\mathrm{CH}_{2}\right) ; 4.11(1 \mathrm{H}, \mathrm{s}, 2-\mathrm{OH})$; ${ }^{13} \mathrm{C} \mathrm{NMR}\left(\mathrm{CDCl}_{3}, 400 \mathrm{MHz}\right) \delta 23.15$ (C-5/C-6), 43.40 (C-3), 80.44 (C-2), 87.23 (C-4), 182.11 (C-1); LC-MS $m / z: 130$.

Compound 3 (2,4-dihydroxy-2,5-dimethylfuran$3(2 \mathrm{H})$-one), pale greenish crystals, molecular formula: $\mathrm{C}_{6} \mathrm{H}_{8} \mathrm{O}_{4}$; yield: $200 \mathrm{mg} ; \mathrm{R}_{\mathrm{f}}: 0.4$ (hexane:ethyl acetate, 1:4); melting point: $163-164^{\circ} ;{ }^{1} \mathrm{H}$ NMR $\left(\mathrm{CDCl}_{3}, 400\right.$ $\mathrm{MHz}) \delta 1.87\left(3 \mathrm{H}, \mathrm{s}, 6-\mathrm{CH}_{3}\right), 2.05\left(3 \mathrm{H}, \mathrm{s}, 5-\mathrm{CH}_{3}\right), 3.62$ $(1 \mathrm{H}, \mathrm{s}, 3-\mathrm{OH}), 3.92(1 \mathrm{H}, \mathrm{s}, 1-\mathrm{OH}) ;{ }^{13} \mathrm{C} \mathrm{NMR}\left(\mathrm{CDCl}_{3}\right.$, $400 \mathrm{MHz}) \delta 12.14$ (C-6), 22.41 (C-5), 99.46 (C-1), 139.32 (C-3), 159.75 (C-4), 192.74 (C-2); CHNS analysis: found C-50.00, H-5.60 (\%), calcd. C, 50.46, H, 5.54 (\%); LC-MS m/z: 144.

\section{Gas chromatography-mass spectrometry (GC-MS) analysis:}

The phytochemical investigation of ethanol extract of C. baltica was performed on a GC-MS system (GCMSQP2010 Plus, Shimadzu, Europe). Experimental parameters set for the GC-MS were, column oven temperature- $50^{\circ}$; injection temperature- $250^{\circ}$; injection mode- split; flow control mode- linear velocity; pressure- $29.7 \mathrm{kPa}$; total flow- $7.3 \mathrm{ml} / \mathrm{min}$; column flow- $0.72 \mathrm{ml} / \mathrm{min}$; linear velocity- $30.7 \mathrm{~cm} / \mathrm{s}$; purge flow- $3.0 \mathrm{ml} / \mathrm{min}$; split ratio- 5.0 ; oven temperature program- $50^{\circ}$ with rate of increase of $10^{\circ} / \mathrm{min}, 50-300^{\circ}$ with rate of increase of $7.5^{\circ} \mathrm{min}$, hold time of $2.0 \mathrm{~min}$; equilibrium time- $1.0 \mathrm{~min}$; ion source temperature$210^{\circ}$; interface temperature- $250^{\circ}$; solvent cut time$3.0 \mathrm{~min}$; detector gain- $0.99+0.20 \mathrm{kV}$; threshold- 1000 ; start time- $4.0 \mathrm{~min}$; end time- $37.30 \mathrm{~min}$; ACQ modescan; event time- $0.50 \mathrm{~s}$; scan speep- 2500; start $\mathrm{m} / \mathrm{z}$ 34.0 and end $\mathrm{m} / \mathrm{z}-1090.00$.

\section{Cytotoxicity assay:}

MCF-7 (breast), DLD-1 (colon), HeLa (cervical), FADU (head and neck), A549 (lung), SKOV3 (ovary) and normal human mammary epithelial (NHME) were kindly provided by National Centre for Cell Science, Pune. The cancer cells were maintained in minimum essential medium (MEM), containing $10 \%$ fetal calf serum (FBS), $5 \%$ mixture of 100 units penicillin and $100 \mu \mathrm{g} / \mathrm{ml}$ streptomycin in presence of $5 \%$ carbon 
dioxide in an incubator with $90 \%$ humidity at $37^{\circ}$ for $72 \mathrm{~h}$.

Cancer cell lines were maintained in MEM (adjusted to $10 \% \mathrm{FBS}, 1.5 \mathrm{~g} / \mathrm{ml} \mathrm{NaHCO}_{3}, 0.1 \mathrm{mM}$ MEM nonessential amino acids and $1 \mathrm{mM}$ sodium pyruvate). Three days prior to performing assay, the cells were washed with sterilized phosphate-buffered saline and grown using MEM media (supplemented with $0.25 \%$ trypsin in versene-EDTA and $10 \% \mathrm{FBS}$ ) and mixed to obtain homogeneous suspension of cells. The suspension was taken in a sterilized polypropylene tube and the cell count in each well was determined in a hemacytometer chamber under a microscope using $0.4 \%$ trypan blue solution. The minimal seed density must be $1.9 \times 10^{4}$ cells per well. All crude extracts and the standard were dissolved in dimethyl sulfoxide (DMSO) to $100 \mathrm{mg} / \mathrm{ml}$ and $10 \mu \mathrm{g} / \mathrm{ml}$, respectively. Doxorubicin and DMSO were used as a standard and control, respectively.

The sulforhodamine B (SRB) assay ${ }^{[13]}$ is based on the estimation of cellular protein content. The prepared samples were taken in 96-well tissue-culture plate and added $190 \mu 1$ screened ideal cell suspension and mixed occasionally and incubate at $37^{\circ}$ with $5 \% \mathrm{CO}_{2}$ and $90 \%$ relative humidity for $3 \mathrm{~h}$. Then $100 \mu \mathrm{l}$ cold TCA was added to each well and incubated at $4^{\circ}$ for $1 \mathrm{~h}$. After that the plates were gently washed using water, dried using blow dryer and air-dried at room temperature. To each completely dried well, $100 \mu 1$ of $0.057 \%$ SRB solution was added, kept aside for 30 min and quickly rinse with $1 \%$ acetic acid. To the dried plate, $200 \mu 1$ of $10 \mathrm{mM}$ Tris base (pH 10.5) solution was added, shake for $5 \mathrm{~min}$ and measure the OD at $510 \mathrm{~nm}$. The blank contains only medium while the control has only cancer cells with no test samples. Percent growth inhibition was calculated using the formula, growth inhibition $(\%)=100-[(\mathrm{S}-\mathrm{B}) /(\mathrm{C}-\mathrm{B})] \times 100$, where $\mathrm{S}$ is mean $\mathrm{OD}$ value of sample, $\mathrm{B}$ is mean OD value of blank, $\mathrm{C}$ is mean OD value of control.

\section{RESULTS AND DISCUSSION}

After the successful extraction of the whole marine algae material with ethanol-water, the dried ethanol extract was subjected to GC-MS analysis. The GCMS chromatogram revealed the presence of various chemical components with different retention times, whereas the MS analyzes the compounds eluted at different times to detect its nature and structure of the compounds. The results pertaining to GC-MS analysis of the ethanol extract lead to the identification of a number of compounds. A total of 86 compounds were identified by GC-MS analysis of ethanol extract, which were tabulated in Table 1.

The collected seaweed was extracted with ethanolwater at room temperature. The obtained ethanol extract $C$. baltica was subjected to chromatographic purification led to isolation of three metabolites (1-3, fig. 1). Compound 1 was obtained as a colorless crystals with $\mathrm{R}_{\mathrm{f}}$ value 0.7 (hexane:ethyl acetate, 1:9) and the LC-MS ion peak at $\mathrm{m} / \mathrm{z} 100$ confirmed the molecular formula as $\mathrm{C}_{4} \mathrm{H}_{4} \mathrm{O}_{3}$. The ${ }^{1} \mathrm{H}$ and ${ }^{13} \mathrm{C}$ NMR data showed the presence of two methane groups at $\delta_{\mathrm{H}} 4.00(s, 4 \mathrm{H})$ with corresponding carbon signal at $\delta_{\mathrm{C}}$ 22.16 (C-2 and C-3). In addition, the ${ }^{13} \mathrm{C}$ NMR also revealed the presence of dione groups at $\delta_{\mathrm{C}} 170.24$ (C-1 and C-4). Hence, compound 1 was confirmed after corroboration with the existing literature ${ }^{[14]}$ as a dihydrofuran-2,5-dione (fig. 1).

Compound 2 was obtained as a pale greenish liquid. Based on the LC-MS ion peak at $m / z 130$ confirmed the molecular formula as $\mathrm{C}_{6} \mathrm{H}_{10} \mathrm{O}_{3}$. The ${ }^{1} \mathrm{H}$ NMR showed the presence of two singlets for two methyl groups at $\delta_{\mathrm{H}} 1.00(\mathrm{~s}, 6 \mathrm{H})$ with corresponding carbon signal at $\delta_{\mathrm{C}}$ 23.15 (C-5 and C-6), including one methine group at $\delta_{\mathrm{H}} 2.31(s, 1 \mathrm{H})$ with corresponding carbon signal at $\delta_{\mathrm{C}}$ $80.44(\mathrm{C}-2)$, and one hydroxyl group at $\delta_{\mathrm{H}} 4.11(s, 1 \mathrm{H})$, additionally, two doublets at $\delta_{\mathrm{H}} 3.86-3.88(d, J=8 \mathrm{~Hz}$, $1 \mathrm{H})$ and $4.04-4.06(d, J=8 \mathrm{~Hz}, 1 \mathrm{H})$, which confirmed the presence of methane group at $\delta_{\mathrm{C}} 87.23$. In addition, the ${ }^{13} \mathrm{C}$ NMR also revealed the presence of dione group at $\delta_{\mathrm{C}} 182.11(\mathrm{C}-1)$. By corroboration with the existing literature compound 2 was found to be 3-hydroxy-4,4dimethyldihydrofuran-2(3H)-one (fig. 1) ${ }^{[15]}$.

Compound 3 was obtained as a pale greenish crystals, based on the LC-MS ion peak at $m / z 144$ confirmed the molecular formula as $\mathrm{C}_{6} \mathrm{H}_{8} \mathrm{O}_{4}$. The ${ }^{1} \mathrm{H}$ NMR showed the presence of four singlets for two methyl groups at $\delta_{\mathrm{H}} 1.87(s, 3 \mathrm{H})$ and $2.05(s, 3 \mathrm{H})$, and for two hydroxyl groups at $\delta_{\mathrm{H}} 3.62(s, 1 \mathrm{H})$ and $3.92(s, 1 \mathrm{H})$. The ${ }^{13} \mathrm{C}$ NMR revealed the presence of two methyl group at $\delta_{\mathrm{C}}$ $12.14(\mathrm{C}-6)$ and $22.41(\mathrm{C}-5)$, two hydroxyl groups at $\delta_{\mathrm{C}}$ $99.46(\mathrm{C}-1)$ and $139.32(\mathrm{C}-3)$, dione group at $\delta_{\mathrm{C}} 192.74$ $(\mathrm{C}-2)$, and one methene carbon signal at $\delta_{\mathrm{C}} 159.75$ (C-4). Based on the aforementioned data, compound 3 was compared with the existing literature on lichen metabolites and it was confirmed as a 2,4-dihydroxy2,5-dimethylfuran-3(2H)-one (fig. 1). Compound 3 was earlier reported from Sysepalum dulcificum ${ }^{[16]}$. 


\begin{tabular}{|c|c|c|c|}
\hline $\begin{array}{l}\text { S. } \\
\text { No }\end{array}$ & Compound name & Chemical structure & Molecular formula \\
\hline 1 & $\begin{array}{c}\text { (2Z)-1,3-dimethyl-6-oxo-2-hexenyl } \\
\text { acetate }\end{array}$ & & $\mathrm{C}_{10} \mathrm{H}_{16} \mathrm{O}_{3}$ \\
\hline 2 & (2Z)-5-methyl-2-decene & & $\mathrm{C}_{11} \mathrm{H}_{12}$ \\
\hline 3 & (9E)-9-octadecenal & & $\mathrm{C}_{18} \mathrm{H}_{34} \mathrm{O}$ \\
\hline 4 & (9Z)-9-octadecenamide & & $\mathrm{C}_{18} \mathrm{H}_{35} \mathrm{NO}$ \\
\hline 5 & $\begin{array}{l}\text { 1-((E)-[(2-methylphenyl)imino }] \\
\text { methyl)-2-naphthol }\end{array}$ & & $\mathrm{C}_{18} \mathrm{H}_{15} \mathrm{NO}$ \\
\hline 6 & $\begin{array}{l}\text { 1,2,5-trimethyl-4-piperidinone } \\
\text { thiosemicarbazone }\end{array}$ & & $\mathrm{C}_{9} \mathrm{H}_{18} \mathrm{~N}_{4} \mathrm{~S}$ \\
\hline 7 & 1,3-cyclohexanedione & & $\mathrm{C}_{6} \mathrm{H}_{8} \mathrm{O}_{2}$ \\
\hline 8 & $\begin{array}{l}\text { 1,4-dimethyl-5-oxabicyclo[2.1.0] } \\
\text { pentane }\end{array}$ & & $\mathrm{C}_{6} \mathrm{H}_{10} \mathrm{O}$ \\
\hline 9 & $\begin{array}{l}\text { 1,5-anhydro-6-deoxyhexo-2,3- } \\
\text { diulose }\end{array}$ & & $\mathrm{C}_{7} \mathrm{H}_{10} \mathrm{O}_{3}$ \\
\hline 10 & 10-undecen-1-ylester & & $\mathrm{C}_{17} \mathrm{H}_{32} \mathrm{O}_{2}$ \\
\hline 11 & 1-chloro-4-decyne & & $\mathrm{C}_{10} \mathrm{H}_{17} \mathrm{Cl}$ \\
\hline 12 & $\begin{array}{l}\text { 1-hexadecylpyridinium chloride } \\
\text { monohydrate }\end{array}$ & & $\mathrm{C}_{21} \mathrm{H}_{40} \mathrm{CINO}$ \\
\hline 13 & 1-Tetradecanol, acetate & & $\mathrm{C}_{16} \mathrm{H}_{32} \mathrm{O}_{2}$ \\
\hline 14 & 1-undecene & & $\mathrm{C}_{11} \mathrm{H}_{22}$ \\
\hline 15 & $\begin{array}{c}\text { 3-hydroxy-4,4- } \\
\text { dimethyldihydrofuran-2(3H)-one }\end{array}$ & & $\mathrm{C}_{6} \mathrm{H}_{10} \mathrm{O}_{3}$ \\
\hline 16 & $\begin{array}{l}\text { 2-(benzyloxy)-4-bromo-1,3- } \\
\text { butanediol }\end{array}$ & & $\mathrm{C}_{11} \mathrm{H}_{15} \mathrm{BrO}_{3}$ \\
\hline 17 & 2,10-dimethylundecane & & $\mathrm{C}_{13} \mathrm{H}_{28}$ \\
\hline 18 & 2,2,4-trimethyl-1-pentanol & & $\mathrm{C}_{8} \mathrm{H}_{18} \mathrm{O}$ \\
\hline 19 & 2,2-dimethyl-1-phenyl-1-propanol & & $\mathrm{C}_{11} \mathrm{H}_{16} \mathrm{O}$ \\
\hline
\end{tabular}


2,2-dioctyl-1-oxohydrazine-1-oxide

9,12-Octadecadienoic acid (Z,Z)-, 2,3-bis(acetyloxy)propyl ester

2,3-dihexyl-2-cyclopropene-1carboxylic acid

\section{2,4,6-trimethyldecane}

2,4-Dihydroxy-2,5-dimethyl-3(2H)furanone

2,5-furandione

2-acetoxytetradecane

2-acetoxytridecane

2-cyclohexen-1-one

\section{2-cyclohexylethyl acetate}

2-hydroxy-3-methylbutanoic acid

2-propylheptan-1-ol

3-(methoxymethoxy)-butanoic acid

3,5,5-trimethyl-4-(3-oxo-1butenyl)-2-cyclohexen-1-one

3,5-bis(1,1-dimethylethyl)-phenol

3,6-dimethyl-2-octanone

3-acetoxydodecane

3-ethyl-2,5-dimethylhexane

3-hexene-2,5-diol

3-hydroxy-2,2,6trimethylcyclohexyl-methyl acetate
$\mathrm{O}_{-\mathrm{N}^{+}} \mathrm{O}$

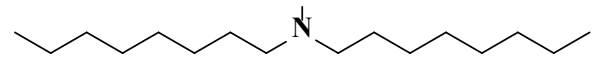

$\mathrm{C}_{16} \mathrm{H}_{34} \mathrm{H}_{2} \mathrm{O}_{2}$<smiles>CC(=O)O</smiles>
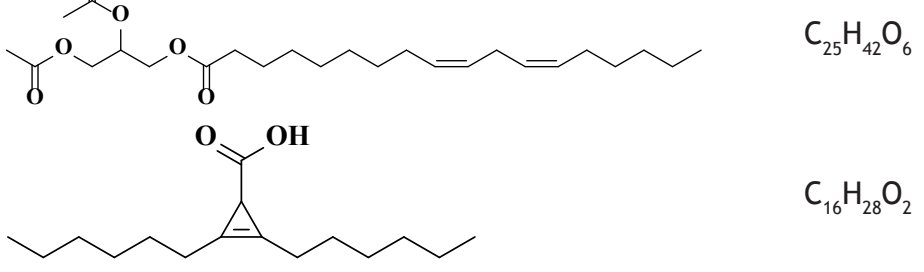

$\mathrm{C}_{16} \mathrm{H}_{28} \mathrm{O}_{2}$

$\mathrm{C}_{13} \mathrm{H}_{28}$<smiles>CC1=C(C)C(=O)C(C)(O)O1</smiles>

$\mathrm{C}_{6} \mathrm{H}_{8} \mathrm{O}_{4}$

$\mathrm{O}^{\mathrm{O}} \mathrm{Y}^{\mathrm{o}}$

$\mathrm{C}_{4} \mathrm{H}_{2} \mathrm{O}_{3}$

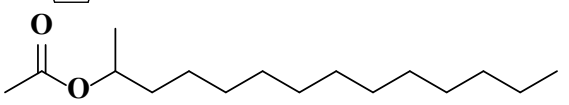

$\mathrm{C}_{16} \mathrm{H}_{32} \mathrm{O}_{2}$<smiles>CCOC(=O)CCCCCOC(C)=O</smiles>

$\mathrm{C}_{15} \mathrm{H}_{30} \mathrm{O}_{2}$

$\mathrm{C}_{6} \mathrm{H}_{8} \mathrm{O}$<smiles>CC(=O)OCCC1CCCCC1</smiles>

$\mathrm{C}_{10} \mathrm{H}_{18} \mathrm{O}_{2}$<smiles>CC(C)C(O)C(=O)O</smiles>

$\mathrm{C}_{5} \mathrm{H}_{10} \mathrm{O}_{3}$<smiles>CCCCCC(CO)CCC</smiles>

$\mathrm{C}_{10} \mathrm{H}_{22} \mathrm{O}$<smiles>COCOC(C)CC(=O)O</smiles>

$\mathrm{C}_{6} \mathrm{H}_{12} \mathrm{O}_{4}$<smiles>CC(=O)/C=C/C1C(C)=CC(=O)CC1(C)C</smiles>

$\mathrm{C}_{13} \mathrm{H}_{18} \mathrm{O}_{2}$<smiles>CC(C)(C)c1cc(O)cc(C(C)(C)C)c1</smiles>

$\mathrm{C}_{14} \mathrm{H}_{22} \mathrm{O}$<smiles>CCC(C)CCCC(C)C(C)=O</smiles>

$\mathrm{C}_{10} \mathrm{H}_{20} \mathrm{O}$

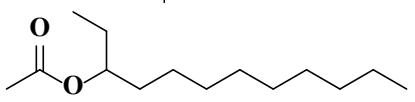

$\mathrm{C}_{14} \mathrm{H}_{28} \mathrm{O}_{2}$<smiles>CCC(CC(C)C)C(C)C</smiles>

$\mathrm{C}_{10} \mathrm{H}_{22}$

$\mathrm{OH}$

$\overbrace{\mathrm{OH}}$

$\mathrm{C}_{6} \mathrm{H}_{12} \mathrm{O}_{2}$

年次 
3-hydroxydodecanoic acid

3-nonyn-1-ol

$$
\text { 4-acetoxypentadecane }
$$

4-heptanol

5-(1-benzyl-1h-indol-3ylmethylene)-1-(2-ethyl-phenyl)pyrimidine-2,4,6-trione

5-bromo-3-methylpentyl acetate

5-cyclopropylidene-1-pentanol

7,9-ditert-butyl-1-oxaspiro[4.5] deca-6,9-diene-2,8-dione

9-octadecenamide

9-octadecenoic acid

Acetic acid

Araldite

Benzenemethanol

Benzoic acid

Butanoic acid

Cis-oleic acid

Cyclopentaneundecanoic acid

Dimethyl(ethenyl)propoxysilane

Di-n-butyl sulphite

Di-n-octyl phthalate

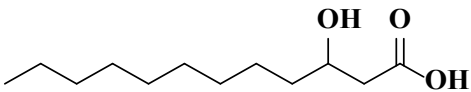

$$
\mathrm{C}_{12} \mathrm{H}_{24} \mathrm{O}_{3}
$$$$
\mathrm{C}_{9} \mathrm{H}_{16} \mathrm{O}
$$$$
\text { o }
$$$$
\mathrm{C}_{17} \mathrm{H}_{34} \mathrm{O}_{2}
$$$$
\mathrm{C}_{7} \mathrm{H}_{16} \mathrm{O}
$$
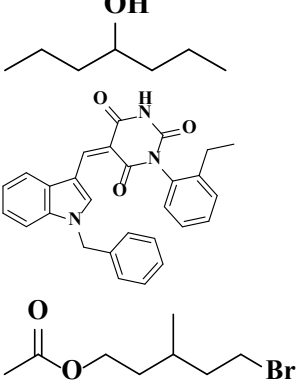

$\mathrm{C}_{8} \mathrm{H}_{15} \mathrm{BrO}_{2}$

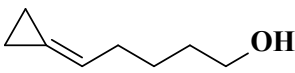

$\mathrm{C}_{8} \mathrm{H}_{14} \mathrm{O}$<smiles>CC(C)(C)C1=CC2(C=C(C(C)(C)C)C1=O)CCC(=O)O2</smiles>

$\mathrm{C}_{17} \mathrm{H}_{24} \mathrm{O}_{3}$<smiles>CCCCCCCC/C=C/CCCCCCCC(N)=O</smiles><smiles>CCCCCCCC/C=C/CCCCCCCC(=O)O</smiles>

点

$\mathrm{C}_{2} \mathrm{H}_{4} \mathrm{O}_{2}$<smiles>O=C1OCCCCCCCCOC(=O)c2ccccc21</smiles>

$\mathrm{C}_{16} \mathrm{H}_{22} \mathrm{O}_{4}$<smiles>OCc1ccccc1</smiles>

$\mathrm{C}_{7} \mathrm{H}_{8} \mathrm{O}$<smiles>O=C(O)c1ccccc1</smiles>

$\mathrm{C}_{7} \mathrm{H}_{6} \mathrm{O}_{2}$

$\mathrm{C}_{4} \mathrm{H}_{8} \mathrm{O}_{2}$<smiles>CCCC(=O)O</smiles>

$\mathrm{C}_{18} \mathrm{H}_{34} \mathrm{O}_{2}$

$\mathrm{C}_{16} \mathrm{H}_{30} \mathrm{O}_{2}$

$\mathrm{C}_{7} \mathrm{H}_{16} \mathrm{OSi}$

$\mathrm{C}_{8} \mathrm{H}_{18} \mathrm{O}_{3} \mathrm{~S}$<smiles>CCCCOSOCCCC</smiles><smiles>C=C(OCCCCCCCCC)c1ccccc1C(=O)OCCCCCCCC</smiles>

$\mathrm{C}_{24} \mathrm{H}_{38} \mathrm{O}_{4}$ 
E-2-undecen-1-ol

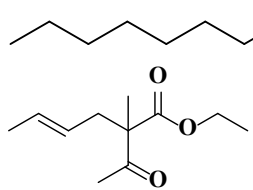

$\mathrm{OH}$

$\mathrm{C}_{11} \mathrm{H}_{22} \mathrm{O}$

Ethyl-(4E)-2-acetyl-2-methyl-4hexenoate

Ethyl docosanoate

Ethyl nonadecanoate

Hexadecyl acetate

Methyl-(E)-docos-13-enoate

Methyl-(9E)-9-octadecenoate

Methyl-3-(3,5-di-tert-butyl-4hydroxyphenyl)propionate

Methyl-3-isopropyl-6-oxoheptanoate Methyl-4-hydroxy-3-methyl-2butenoate

Methyl heptanoate

n-cyclohexyl methacrylamide

n-decyl acetate

n-heptadecane

Octadecane

Octadecanoic acid

Octanal dimethyl acetal

Palmitic acid

$$
\text { Palmitic acid ethyl ester }
$$

Pelargonic acid methyl ester

$$
\text { Pentanoic acid }
$$

Phthalic acid

Tert-nonanethiol
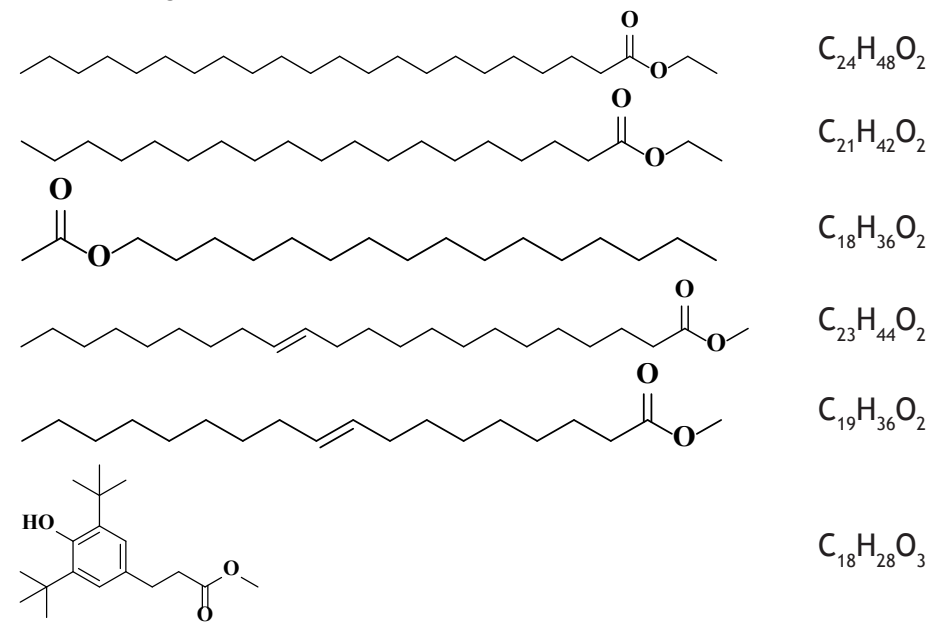

$\mathrm{C}_{19} \mathrm{H}_{36} \mathrm{O}_{2}$

$\mathrm{C}_{18} \mathrm{H}_{28} \mathrm{O}_{3}$<smiles>COC(=O)CC(CCC(C)=O)C(C)C</smiles>

$\mathrm{C}_{11} \mathrm{H}_{20} \mathrm{O}_{3}$<smiles>COC(=O)/C=C(\C)CO</smiles><smiles>CCCCCCC(=O)OC</smiles><smiles>C=C(C)C(=O)NC1CCCCC1</smiles>

$\mathrm{C}_{10} \mathrm{H}_{17} \mathrm{NO}$

O

$\mathrm{C}_{12} \mathrm{H}_{24} \mathrm{O}_{2}$

$\mathrm{C}_{17} \mathrm{H}_{36}$<smiles>CCCCCCCCCCCCCCCCCCCCCCCC</smiles><smiles>CCCCCCCCCCCCCCCCCC(=O)O</smiles>

$\mathrm{C}_{10} \mathrm{H}_{22} \mathrm{O}_{2}$

$\mathrm{C}_{16} \mathrm{H}_{32} \mathrm{O}_{2}$

$\mathrm{C}_{18} \mathrm{H}_{36} \mathrm{O}_{2}$

$\mathrm{C}_{10} \mathrm{H}_{20} \mathrm{O}_{2}$

$\mathrm{C}_{5} \mathrm{H}_{10} \mathrm{O}_{2}$

$\mathrm{C}_{8} \mathrm{H}_{6} \mathrm{O}_{4}$

$\mathrm{C}_{9} \mathrm{H}_{20} \mathrm{~S}$ 
85

86
Tetradecane

Xanthosine

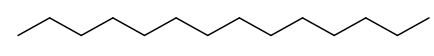

$\mathrm{C}_{14} \mathrm{H}_{30}$

OH

HO

OOH

TABLE 2: CYTOTOXICITY STUDIES OF COMPOUNDS 1-3 AND ETHANOL EXTRACT OF CHARA BALTICA

\begin{tabular}{cccccccc}
\hline Sample & \multicolumn{7}{c}{ Percent growth inhibition at $30 \mu g / m l$} \\
\hline 1 & MCF-7 & DLD-1 & HeLa & FADU & A549 & SKOV3 & NHME $^{c}$ \\
\hline & $1.46 \pm 0.18$ & $5.64 \pm 0.65$ & $3.97 \pm 0.70$ & $13.80 \pm 1.06$ & $21.37 \pm 1.94$ & $5.69 \pm 0.87$ & $0.52 \pm 0.01$ \\
3 & $7.17 \pm 0.24$ & $11.91 \pm 0.50$ & $5.12 \pm 0.18$ & $16.41 \pm 1.26$ & $14.57 \pm 0.39$ & $8.14 \pm 1.05$ & $1.04 \pm 0.16$ \\
Ethanol extract $^{*}$ & $56.86 \pm 4.78$ & $76.37 \pm 5.34$ & $22.27 \pm 6.23$ & $64.08 \pm 3.92$ & $34.07 \pm 4.84$ & $46.11 \pm 4.74$ & $9.40 \pm 0.33$ \\
Doxorubicin $^{*}$ & $84.40 \pm 0.80$ & $66.71 \pm 0.71$ & $90.71 \pm 0.13$ & $88.34 \pm 0.27$ & $65.40 \pm 0.60$ & $77.05 \pm 0.22$ & $10.08 \pm 0.95$ \\
\hline
\end{tabular}

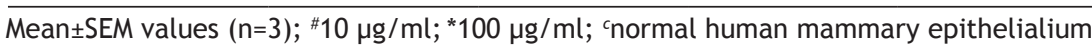

TABLE 3: GROWTH INHIBITION AND IC ${ }_{50}$ VALUES OF COMPOUND 3 AND ETHANOL EXTRACTS OF CHARA BALTICA

\begin{tabular}{|c|c|c|c|c|c|}
\hline \multirow{2}{*}{ Cell line sample } & \multicolumn{4}{|c|}{ Concentration $(\mu \mathrm{g} / \mathrm{ml})$} & \multirow{2}{*}{$\begin{array}{c}\mathrm{IC}_{50} \text { values } \\
(\mu \mathrm{g} / \mathrm{ml})\end{array}$} \\
\hline & 5 & 10 & 20 & 30 & \\
\hline \multicolumn{6}{|l|}{ MCF-7 } \\
\hline Compound 3 & $18.09 \pm 2.87$ & $28.17 \pm 2.87$ & $38.59 \pm 1.74$ & $56.86 \pm 4.78$ & 26.5 \\
\hline Ethanol extracts** & $25.06 \pm 1.78$ & $36.58 \pm 2.68$ & $50.05 \pm 3.57$ & $71.53 \pm 4.71$ & 75 \\
\hline Doxorubicin* & $30.87 \pm 0.61$ & $45.45 \pm 0.18$ & $67.08 \pm 0.11$ & $81.25 \pm 0.27$ & 5.5 \\
\hline \multicolumn{6}{|l|}{ DLD-1 } \\
\hline Compound 3 & $22.55 \pm 4.84$ & $39.77 \pm 2.83$ & $57.92 \pm 7.78$ & $76.37 \pm 5.34$ & 15.5 \\
\hline Ethanol extract ${ }^{* *}$ & $29.75 \pm 1.77$ & $42.57 \pm 2.49$ & $59.22 \pm 1.77$ & $87.11 \pm 3.58$ & 61 \\
\hline Doxorubicin* & $19.75 \pm 4.61$ & $31.58 \pm 6.85$ & $44.75 \pm 2.84$ & $52.57 \pm 7.97$ & 5.4 \\
\hline \multicolumn{6}{|l|}{ FADU } \\
\hline Compound 3 & $29.68 \pm 2.68$ & $40.88 \pm 4.06$ & $54.87 \pm 4.54$ & $64.08 \pm 3.92$ & 16.5 \\
\hline Ethanol extract ${ }^{* *}$ & $26.47 \pm 2.97$ & $37.18 \pm 2.52$ & $50.17 \pm 3.58$ & $70.46 \pm 4.96$ & 74.5 \\
\hline Doxorubicin* & $41.01 \pm 3.85$ & $52.14 \pm 2.84$ & $68.88 \pm 1.77$ & $85.55 \pm 6.24$ & 4.5 \\
\hline \multicolumn{6}{|l|}{ A549 } \\
\hline Ethanol extract ${ }^{* *}$ & $26.47 \pm 2.97$ & $37.18 \pm 2.52$ & $50.17 \pm 3.58$ & $57.62 \pm 5.58$ & 74.5 \\
\hline Doxorubicin* & $41.01 \pm 3.85$ & $52.14 \pm 2.84$ & $68.88 \pm 1.77$ & $85.55 \pm 6.24$ & 4.5 \\
\hline
\end{tabular}

Each value is a mean+SD of \% growth inhibition of the cell line tested with $\mathrm{n}=3 ;{ }^{*} 2.5,5.0,7.5$ and $10 \mu \mathrm{g} / \mathrm{ml}$ concentrations; ** $25,50,75$ and $100 \mu \mathrm{g} / \mathrm{ml}$ concentrations
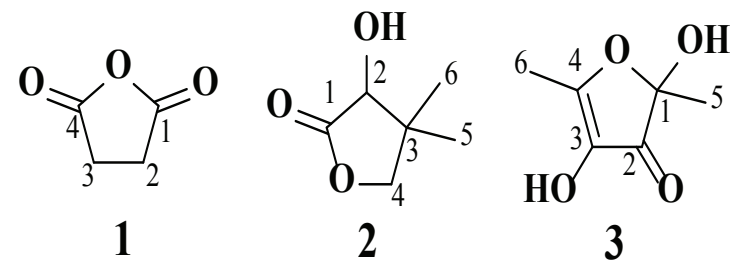

Fig. 1: Known secondary metabolites from ethanol extracts of Chara baltica

Earlier reports of $C$. baltica indicated free radical scavenging activity ${ }^{[13]}$. Based on the report, the isolated metabolites (1-3) were screened along with ethanol extract against six different cancer cell lines using SRB assay with doxorubicin as standard. Initially, compounds (1-3) at $30 \mu \mathrm{g} / \mathrm{ml}$ concentration, extract at $100 \mu \mathrm{g} / \mathrm{ml}$ concentration and doxorubicin at $10 \mu \mathrm{g} / \mathrm{ml}$ concentration were screened against the cell lines tested and percent inhibition of cell growth was tabulated in Table 2.

Further, the samples that caused $50 \%$ or more of cell death were further screened at 5, 10, 20 and $30 \mu \mathrm{g} / \mathrm{ml}$ concentrations for pure compounds; 25, 50, 75 and $100 \mu \mathrm{g} / \mathrm{ml}$ concentrations for extracts; 2.5, 5.0, 7.5 and $10 \mu \mathrm{g} / \mathrm{ml}$ concentrations for doxorubicin. The results were plotted to obtain $\mathrm{IC}_{50}$ values. The lower $\mathrm{IC}_{50}$ value indicated better inhibitory profile against cancer cell lines.

During the initial screening, the isolate 3 and ethanol extract depicted prominent degree of specificity against MCF-7, DLD-1, FADU and A549. Besides, all the samples exhibited very low toxicity towards NHME cell 
lines (Table 2). From the outcomes of the SRB assay, it is interesting to note that compound $3(30 \mu \mathrm{g} / \mathrm{ml})$ and ethanol extract $(100 \mu \mathrm{g} / \mathrm{ml})$ showed prominent degree of specificity against DLD-1 with $76.37 \pm 5.34$ and $87.11 \pm 3.58 \%$ growth inhibition which was found to be better than activity of $66.71 \pm 0.71 \%$ shown by the standard at $10 \mu \mathrm{g} / \mathrm{ml}$ (Table 2).

From the Table 3, the $\mathrm{IC}_{50}$ values of 3 and ethanol extract against MCF-7 were 26.5 and $75.0 \mu \mathrm{g} / \mathrm{ml}$, respectively, whereas standard value was $5.5 \mu \mathrm{g} / \mathrm{ml}$. In addition, the concentration of 3 and ethanol extract needed for $50 \%$ inhibition of DLD-1 were found to be 15.5 and $61.0 \mu \mathrm{g} / \mathrm{ml}$, respectively, while for doxorubicin it was $5.4 \mu \mathrm{g} / \mathrm{ml}$ (Table 3).

From the SRB assay of FADU (Table 3) it can be calculated that the $\mathrm{IC}_{50}$ values of 3 and ethanol extract against FADU were 16.5 and $74.5 \mu \mathrm{g} / \mathrm{ml}$, respectively, whereas standard value was $4.5 \mu \mathrm{g} / \mathrm{ml}$. Additionally, the concentration of ethanol extract needed for $50 \%$ inhibition of A549 was found to be $74.5 \mu \mathrm{g} / \mathrm{ml}$, while standard (doxorubicin) was $4.5 \mu \mathrm{g} / \mathrm{ml}$ (Table 3 ).

Hence, from the overall SRB assay it can be concluded that the key agent responsible for anticancer activity is compound 3. In addition, the growth inhibitory properties of compound 3 is mainly due to the higher levels of oxygenated substituents present in its chemical structure, this higher levels of oxygenated content help in irradiating free radicals that are usually formed during massive cell division of cancer cells, which ultimately lead to the cell death ${ }^{[13,17]}$.

In conclusion, this is the first report of GC-MS analysis, chemical and cytotoxicity profile of marine algae C. baltica. The GC-MS analysis of ethanol extract C. baltica revealed presence of 89 chemical constituents. In addition, the chemical examination of ethanol extract showed the presence of three metabolites 1-3. Additionally, the cytotoxicity studies of ethanol extract indicated specificity towards MCF-7, DLD-1, FADU and A549 without affecting NHME. The SRB assay screening proved that compound 3 has greater cytotoxicity. The results of the current study could be useful for further research on cancer to identify potential bioactive molecules from different aquatic fauna, such as marine algae like $C$. baltica.

\section{Acknowledgement:}

The authors thank the authorities of CDRI-Lucknow, India for providing the cytotoxicity testing facilities to complete present work. The authors wish to thank
Dr. G. Mohan Narasimha Rao, Professor of Botany Department, Andhra University, Visakhapatnam, for authenticating the seaweed.

\section{Conflict of interest:}

No conflict of interest between any of the authors.

\section{REFERENCES}

1. Ochwang'I DO, Kimwele CN, Oduma JA, Gathumbi PK, Mbaria JM, Kiama SG. Medicinal plants used in treatment and management of cancer in Kakamega county Kenya. J Ethnopharmacol 2014;151:1040-55.

2. Bray F, Ferlay J, Soerjomataram I, Siegel RL, Torre LA, Jemal A. Global cancer statistics 2018: GLOBOCAN estimates of incidence and mortality worldwide for 36 cancers in 185 countries. CA Cancer J Clin 2018;68:394-424.

3. Solowey E, Lichtenstein M, Sallon S, Paavilainen H, Solowey E, Lorberboum-Galski H. Evaluating Medicinal Plants for Anticancer Activity. Scientific World J 2014;2014:721402.

4. Greenwell M, Rahman PK. Medicinal plants: their use in anticancer treatment. Int J Pharm Sci Res 2015;6:4103-12.

5. Newman DJ. Natural products as leads to potential drugs: an old process or the new hope for drug discovery. J Med Chem 2008;51(9):2589-99.

6. Newman DJ, Cragg GM, Snader KM. Natural products as sources of new drugs over the period 1981-2002. J Nat Prod 2003;66:1022-37.

7. Val A, Platas G, Basilio A, Cabello A, Gorrochategui J, Suay $\mathrm{I}$, et al. Screening of antimicrobial activities in red, green and brown macroalgae from Gran Canaria (Canary Islands, Spain). Int Microbiol 2001;4(1):35.

8. Patterson GML, Larsen LK, Moore RE. Bioactive natural products from blue-green algae. J Appl Phycol 1994;6:151.

9. Pietra F. Secondary metabolites from marine microorganisms: bacteria, protozoa, algae and fungi. Achievements and prospects. Nat Prod Rep 1997;14(5):453-64.

10. Cabrita MT, Vale C, Rauter AP. Halogenated compounds from marine algae. Mar Drugs 2010;8(8):2301-17.

11. Berger J, Schagerl M. Allelopathic activity of Characeae. Hydrobiologia 2003;501(1-3):109.

12. Naidu KK, Bharadwaj TV, Alekya K, Lakshmi YB, Sastry VG. Qualitative analysis and free radicals scavenging ability of marine algae, Chara baltica. J Integr Sci 2018;1:1-5.

13. Tatipamula VB, Vedula GS, Sastry AVS. Antarvediside A-B from manglicolous lichen Dirinaria consimilis (Stirton) D.D. Awasthi and their pharmacological profile. Asian J Chem 2019;31:805-12.

14. Buechi G, Demole E, Thomas AF. Syntheses of 2,5-dimethyl-4hydroxy-2,3-dihydrofuran-3-one (furaneol), a flavor principle of pineapple and strawberry. J Org Chem 1973;38:123-5.

15. Pansare SV, Shinkre BA, Bhattacharyya A. Enantioselective synthesis of $\alpha$-hydroxy $\gamma$-butyrolactones from an ephedrinederived morpholine-dione. Tetrahedron 2002;58:8985-1.

16. Chukwu CJ, Omaka ON, Aja PM. Characterization of 2,5-dimethyl-2,4-dihydroxy-3(2H) furanone, a flavourant principle from Sysepalum dulcificum. Nat Prod Chem Res 2017;5:8.

17. Shoemaker M, Hamilton B, Dairkee SH, Cohen I, Campbell MJ. In vitro anticancer activity of twelve Chinese medicinal herbs. Phytother Res 2005;19:649-51. 